\title{
El método del caso como herramienta de aprendizaje activo en arqueología
}

Sonia Machause López ${ }^{\text {a }}$, Gianni Gallello ${ }^{\text {, }}$ David Quixal Santos ${ }^{\text {a }}$ Agustín Diez Castillo ${ }^{a}$, Consuelo Mata Parreño a Iván Fumadó Ortega ${ }^{\mathrm{a}}$

aDepartament de Prehistòria, Arqueologia i Història Antiga (Universitat de València). E-mails: sonia.machause@uv.es, gianni.gallello@uv.es, david.quixal@uv.es, agustin.diez@uv.es, consuelo.mata@uv.es, i.fumado.ortega@uv.es

\section{\$EWWFW}

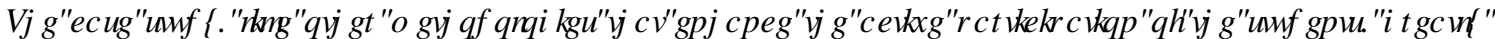

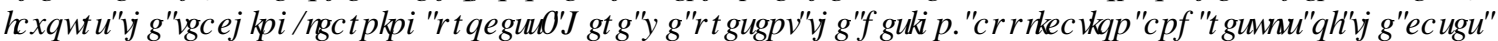

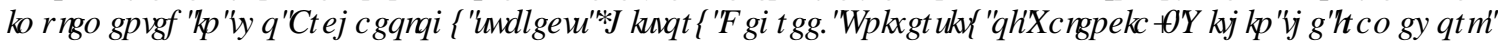

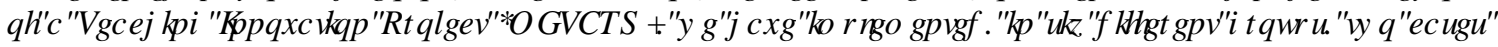

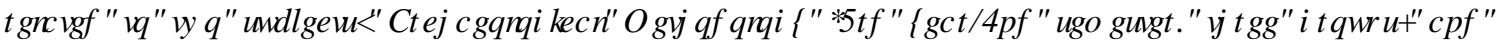

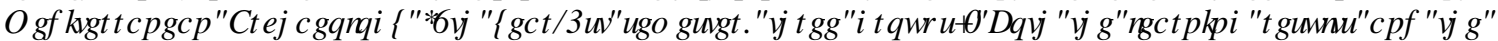

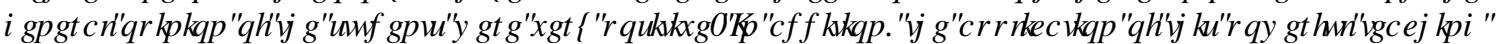

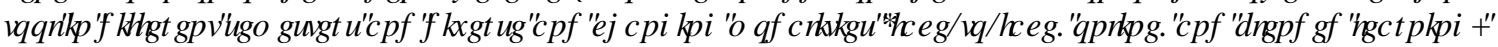

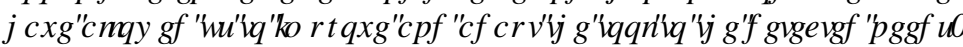
$\square$

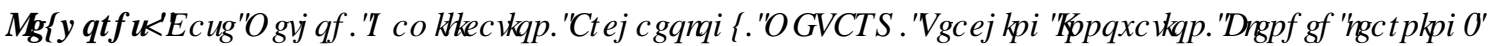

\section{HXP HQD}

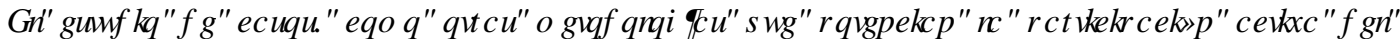

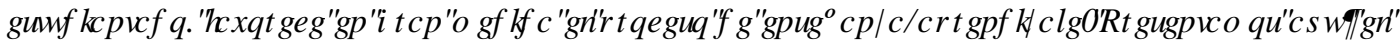

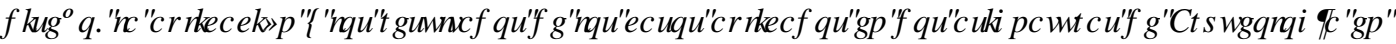

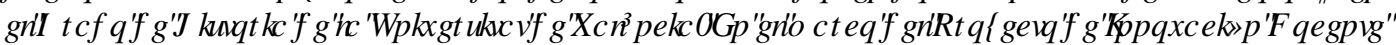

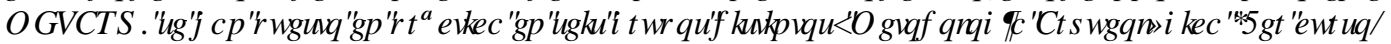

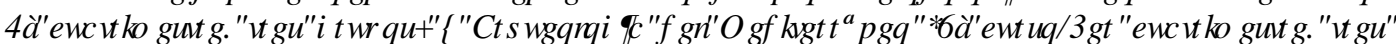

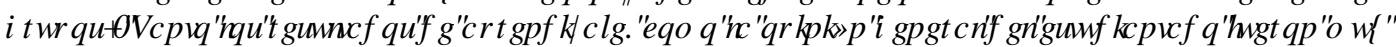

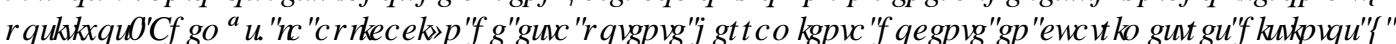

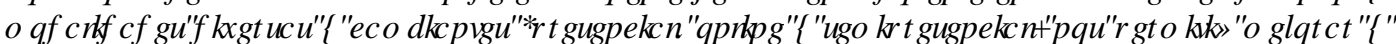

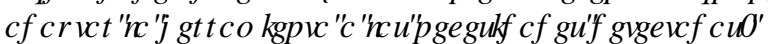

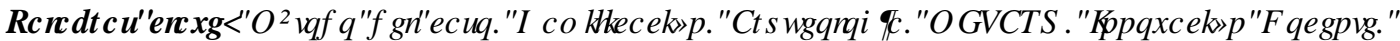
6HP ISUHMQFIDOCDOT 


\section{Introducción}

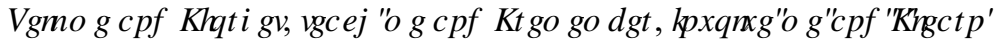

(Benjamin Franklin)

El aprendizaje significativo tan solo se consigue si implicamos al estudiantado y potenciamos su participación activa, estimulando la reflexión y el proceso de aprendizaje a partir de sus propias experiencias. Tal y como indica Finkel (2008: 40), con el paso del tiempo tan solo permanece en la memoria aquello que nos ha hecho pensar y nos ha motivado a buscar respuestas razonables.

En el contexto actual, marcado por la docencia en línea o semipresencial, derivada de la pandemia de la COVID-19, el profesorado universitario se ha visto obligado a adaptar las herramientas docentes a marchas forzadas (Bao $H M D O$, 2020; Gallello $H W D O, 2020$ ). Sin embargo, es posible y necesario activar nuevas estrategias que potencien la motivación del estudiantado y mejoren el aprendizaje significativo, incluso a través de una pantalla.

Atendiendo a las carencias y las necesidades detectadas en cursos previos en los que hemos impartido asignaturas de Arqueología en el Grado de Historia (Universitat de València), nos propusimos activar nuevas formas de aprendizaje que mejoraran los resultados y se adaptaran a diversas realidades. La iniciativa que presentamos aquí forma parte del Proyecto de Innovación Docente 0 ( 7\$54 एO HRGRQRTDV

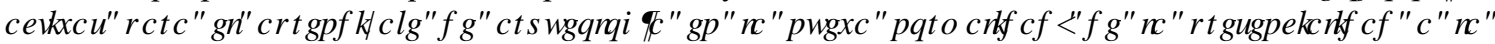
VP ISUHMPFIDOCDG I

La puesta en práctica del método de caso se ha llevado a cabo en las asignaturas de Metodología Arqueológica ( $2^{\circ}$ cuatrimestre/3er curso, 3 grupos: dos en horario de mañana -castellano y valenciano- y uno de tarde -castellano-) y Arqueología del Mediterráneo (1er cuatrimestre $/ 4^{\circ}$ curso, 3 grupos: dos en horario de mañana -castellano y valenciano- y uno de tarde -castellano-). El equipo docente de ambas asignaturas comparte la preocupación frente a la falta de participación, el abandono temprano y la dificultad para comprender conceptos novedosos. Desgraciadamente, los cambios en los planes de estudios derivados del Plan Bolonia y la reducción de materias relacionadas con la Arqueología en el actual Grado de Historia de la Universitat de València, son una de las causas principales de este desinterés y falta de conocimientos teórico-prácticos. Hace una decena de años, con la Licenciatura de Historia, estas carencias no eran tan pronunciadas, en gran parte por la variedad de asignaturas obligatorias y optativas relacionadas con la Arqueología disponibles en los planes antiguos ${ }^{2}$. Los conceptos que hace unos años se adquirían progresivamente en distintas asignaturas, actualmente se concentran en dos asignaturas obligatorias (3er y $4^{\circ}$ curso) y una asignatura optativa (Arqueología de la Península Ibérica: $4^{\circ}$ curso). Si bien algunos conceptos comunes se introducen en asignaturas de Prehistoria durante los primeros cursos del grado, estos no son suficientes. En este contexto, el equipo docente de las asignaturas de Arqueología centramos nuestros esfuerzos en potenciar la motivación y mejorar el proceso de enseñanza-aprendizaje con los recursos y el tiempo limitado que disponemos para impartir nuestras materias. Debemos tener en cuenta, además, que es durante los primeros cursos cuando el estudiantado, generalmente, escoge qué especialización seguirá en el grado (antes de haber cursado asignaturas de Arqueología). Por ello mismo pensamos que es todavía más necesario mostrar esta disciplina de una forma atractiva y cautivadora. En este artículo nos centramos en una de las metodologías, desarrolladas en el marco del proyecto METARQ, que ha sido mejor valorada por el profesorado y estudiantado implicado: el método del caso.

\footnotetext{
${ }^{1}$ Concedido en el marco de los Proyectos de Innovación Docente (NOU-PID) 2020/21 de la Universitat de València (UV-SFPIE_PID-1355034). Agradecemos al Vicerrectorat d'Ocupació Permanent/SFPIE la concesión del proyecto, así como a la totalidad de miembros del mismo que están colaborando en distintas actividades de innovación docente durante el curso 2020-2021.

${ }^{2}$ Asignaturas como Arqueología Ibérica, Arqueología Fenicio-Púnica, Arqueología del Mar, Arqueología de los Animales, Arqueología Clásica, Arqueología del Territorio, Prácticas de Cultura Material Arqueológica, Introducción al Patrimonio Arqueológico Valenciano, entre otras.
} 


\section{Objetivos principales}

Los objetivos específicos de nuestra aplicación del método del caso son:

- Asimilar, comprender y poner en práctica los conceptos adquiridos en las clases teóricas, así como en asignaturas de cursos anteriores.

- Mejorar el trabajo cooperativo, el diálogo y el aprendizaje entre iguales.

- Motivar la participación activa y la asistencia a clase (presencial o virtual).

- Favorecer el aprendizaje autónomo y significativo.

- Promover la organización, la iniciativa y la toma de decisiones.

- Motivar el debate y la expresión de ideas con una argumentación previamente definida de manera colaborativa.

- Desarrollar la creatividad e imaginación para la solución de problemas.

\section{Desarrollo de la innovación}

\subsection{La herramienta del método del caso}

Hace ya más de un siglo y medio que el método del caso se utiliza en distintas disciplinas para desarrollar el aprendizaje a través de problemas. La base de esta herramienta se centra en la experimentación de situaciones realistas o de la vida real, normalmente abordados por pequeños grupos de estudiantes bajo la dirección del profesorado. Esta herramienta se considera la precursora del aprendizaje basado en problemas, que se desarrolló un siglo después. En pocos años el caso se hizo muy popular, sobre todo en el ámbito de los estudios de derecho, siendo ampliamente adoptado en Estados Unidos (Servant-Miklos, 2019) y considerándose un instrumento muy eficaz, atendiendo a diversas esferas (Nkhoma $H M D D$ 2017):

1. La esfera motivacional, que hace hincapié en el compromiso individual y lleva al estudiantado a animarse y ayudarse para lograr las metas comunes en el grupo.

2. La esfera afectiva, que está muy centrada en el rol del profesorado en facilitar la interacción entre el estudiantado.

3. Y la esfera cognitiva que hace que el estudiantado perciba la práctica como una oportunidad para debatir y presentar sus ideas, así como escuchar y reflexionar sobre el punto de vista de los/las compañeros/as.

La eficacia de esta herramienta radica en la premisa de que el aprendizaje depende de la responsabilidad y la actitud del estudiantado. Por tanto, el sujeto no es el profesorado y tampoco el contenido académico, sino el aprendizaje autónomo del estudiantado. Las ventajas del método del caso radican en el impulso de los aspectos motivacionales para mejorar el aprendizaje, la retención de contenidos, la estimulación de la comprensión y la apreciación profunda del tema tratado.

En los últimos años, se han desarrollado diversos trabajos críticos sobre la eficacia de este método de aprendizaje y el uso apropiado que se hace del mismo en el ámbito universitario (ver Kilbane $H W D O$ 2004; Gerring 2007; Liu $H W D O, 2009$; Laru $H W D O$, 2012; Baeten $H W D O$, 2013; Harland, 2014; Massis y Kotlar, 2014; Rashid $H W D O$, 2019; Safapour $H W D O$, 2019, entre otros). En general, estos estudios convergen en subrayar que la eficacia del caso se materializa si surgen conceptos, ideas y debates inesperados, porque es entonces cuando realmente se contribuye al aprendizaje de conocimientos teóricos y prácticos. Una reflexión interesante que pone en evidencia los límites de esta herramienta docente ha sido propuesta por Kreber (2001). Esta autora subraya que proporcionar al estudiantado casos concretos no conduce necesariamente a experiencias de aprendizaje. Tratando contenidos teóricos abstractos, mediante lecturas que preceden las experiencias prácticas, también se consigue potenciar el aprendizaje. El éxito del método del caso depende en gran medida de la responsabilidad del profesorado, encargado de fomentar el aprendizaje experiencial. El profesorado debe, por tanto, animar al estudiantado a transformar conceptos abstractos en experiencias reales, provocando experimentación y reacción. Kreber (2001) analiza también un aspecto fundamental relacionado con las áreas de conocimiento. Generalmente, se piensa que es más fácil trasformar en experiencia conceptos abstractos de las ciencias (p.e. "el efecto invernadero") que contenidos abstractos propios de las ciencias sociales y humanidades. Se cree que estos contenidos (p.e. "el concepto de democracia") es más sencillo convertirlos en respuestas. Sin embargo, en ambos casos se puede aplicar la

(c)) BY-NC-ND 2021, Universitat Politècnica de València

CRQJUHR, Q5 HGHपिए। 
misma herramienta, creando un evento concreto para que sirva como ejemplo de un concepto abstracto, tal y como se ha observado en algunos casos publicados que plantean la aplicación de este método tanto de manera interdisciplinar (en áreas de ecología y ciencias sociales) (Steiner y Posch, 2006), así como en áreas concretas como la geografía (Koç, 2018; Cousin, 2005).

\subsection{Diseño y aplicación de la herramienta

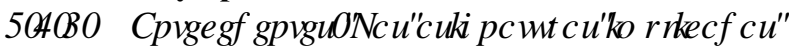

Atendiendo a las necesidades y las carencias detectadas en cursos previos, por parte del equipo docente de las asignaturas de Metodología Arqueológica y Arqueología del Mediterráneo, propusimos una serie de actividades prácticas que facilitaran la comprensión de ciertos conceptos y temas, entre las que se encuentra el método del caso.

La primera asignatura en la que probamos esta herramienta fue Arqueología del Mediterráneo (código 34031). Es una asignatura obligatoria, de 6 ECTS, que forma parte del primer cuatrimestre del cuarto curso en el Grado de Historia de la Universitat de València. El objetivo principal de esta asignatura es la

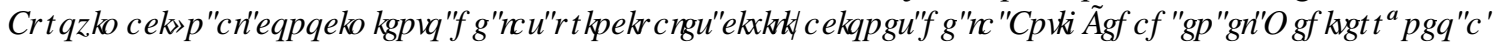
WVYVWGHQRVLHMRVDUXHRQJIFR $V^{8} \square \mathrm{La}$ asignatura se divide en 10 temas: Introducción (T.1), La ciudad (T.2), Territorio (T.3), Urbanismo (T.4), Obras de ingeniería (T.5), Espacio doméstico (T.6), Lugares de culto (T.7), Mundo funerario (T.8), Escultura y pintura (T.9) y Cerámica y joyería (T.10). Debido a la situación derivada de la COVID-19, las clases durante el primer cuatrimestre del curso 2020-2021 siguieron el sistema híbrido, organizado en semanas alternas. Por tanto, el estudiantado de las clases teóricas y prácticas se dividía en dos subgrupos que asistían presencialmente a clase cada 15 días. Sin embargo, tanto las clases teóricas como las prácticas se retransmitían a través de \%OFNERDCT\&RCDERLDU El número de alumnos matriculados durante el curso 2020-2021 varía entre grupos: 73 alumnos (grupo A), 41 alumnos (grupo B) y 68 alumnos (grupo C).

Durante el segundo cuatrimestre se realizó una actividad similar en los tres grupos de la asignatura de Metodología Arqueológica (código 34053: 6 ECTS): grupo A (74 alumnos), grupo B (58 alumnos) y grupo C (52 alumnos). A grandes rasgos, esta asignatura busca la DSLR[IP DFIYQDDQRVP $p$ LRCRVFIHQWIFRVTXH

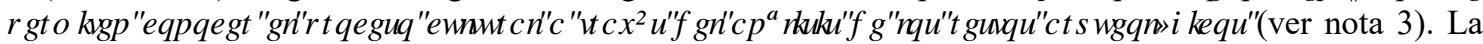
asignatura se divide en 5 temas: ¿Qué es la Arqueología? (T.1), El tiempo (T.2), Arqueología funeraria y el mundo de las ideas (T.3), El paisaje, los recursos y la producción (T.4) y El territorio, el intercambio y el comercio (T.5). Debido a la tercera ola de la pandemia, el inicio del segundo cuatrimestre se realizó completamente online ${ }^{4}$, volviendo al sistema híbrido en marzo.

\section{पषणि ' LMKRIGHLRVIFDRV}

La aplicación de la herramienta implica un amplio trabajo de preparación y planificación previo por parte del profesorado. El papel del equipo docente es vital no solo en el diseño y la preparación de los casos, sino también en el desarrollo y orientación del estudiantado (Wasserman, 1999). Antes de poner en práctica la actividad, nos planteamos las siguientes preguntas clave:

- ¿Qué conceptos son más complejos y difíciles de comprender para el estudiantado?

- ¿Qué objetivos perseguimos con esta actividad?

- ¿Cómo podemos motivar al aprendizaje?

Tras debatir estas cuestiones, diseñamos un problema que permitiera potenciar el aprendizaje de dos temas: las evidencias arqueológicas en las prácticas funerarias greco-romanas (Arqueología del Mediterráneo) y los métodos de datación en Arqueología (Metodología Arqueológica).

En el primer ejemplo, el objetivo principal se centraba en que reflexionaran sobre las evidencias visibles e invisibles en el registro arqueológico y cómo, basándonos en estas evidencias, es posible reconstruir parcialmente un contexto funerario en el mundo clásico. De este modo, se conseguían también objetivos complementarios como, por ejemplo, la reflexión sobre evidencias invisibles (arqueología sensorial, sesgos

\footnotetext{
${ }^{3}$ Plan de estudios del Grado de Historia: https:/www.uv.es/uvweb/grado-historia/es/se-estudia/plan-estudios/plan-estudios/grado-historia-

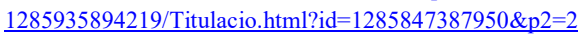

${ }^{4}$ https:/www.uv.es/uvweb/uv-noticies/es/noticias/universidades-publicas-valencianas-acuerdan-generalitat-minima-presencialidad-inicio-docencia-delsegundo-cuatrimestre-1285973304159/Novetat.html?id=1286171820306\&plantilla=UV Noticies/Page/TPGDetaillNews
}

(cc) BY-NC-ND 2021, Universitat Politècnica de València

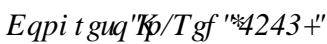


en el registro arqueológico, expolio...), la interdisciplinariedad en arqueología o problemáticas como la falta de financiación en investigación. La atracción se producía gracias al misterio de la aparición de un objeto arqueológico en unas obras urbanas en Atenas (fig. 1) y en Roma. Si bien el problema era similar en ambos ejemplos, las evidencias variaban ligeramente adaptándose a cronologías y ámbitos culturales distintos, para poder realizar la misma actividad en dos semanas correlativas (ver apartado 3.2.3). Teniendo unas evidencias arqueológicas determinadas, pero incompletas, debían consensuar con los miembros de su equipo una solución al problema planteado y enfrentarse a una rueda de prensa.

En el segundo ejemplo, el objetivo principal se centraba en que comprendieran los distintos métodos de datación (relativa y absoluta) y los aplicaran a un problema "real". La implicación y la motivación derivaba de la serie de TVE ( OO LQLUHIR GHD7IHSR , ya que no se trataba de un contexto arqueológico común, sino que había sufrido una alteración temporal (fig. 2). Al tratarse de un contexto funerario, no solo se potenciaba el aprendizaje de cuestiones relativas a la datación para conocer la cronología de la tumba y de los viajes en el tiempo, sino que se generaba interés y conocimiento sobre la cremación, el ritual funerario ibérico y la cultura material asociada (cerámicas ibéricas, cerámicas de importación griega, objetos de bronce, ...). Teniendo en cuenta la cronología aportada por ciertos restos y averiguando la datación del resto de evidencias arqueológicas y actuales, debían consensuar con los miembros de su equipo una cronología aproximada y enfrentarse a las preguntas planteadas por el Ministerio del Tiempo. 


\section{MÉTODO DEL CASO: \\ UNA PILDRA EXTRAÑA EN LA ZANJA}

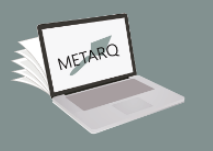

Contexto

Sois un equipo de arqueólogas y arqueólogos que estáis trabajando en Atenas en un seguimiento de unas obras de canalización al noroeste de la ciudad. Los dos primeros días trascurrieron con normalidad. Por suerte, el equipo de peones que os han asignado está trabajando a un ritmo acelerado y, seguramente, podréis acabar antes de que comience la obra que tenéis en el puerto.

Sin embargo, el día de hoy será diferente... A las 10:00h, mientras estabais acabando el croquis de la zona de actuación, el jefe de obra llama vuestra atención diciendo: “Aquí ha aparecido una piedra con forma extraña”. Os desplazáis hasta la zanja y observáis que "la piedra con forma extraña" no es una piedra cualquiera, se trata de una estela funeraria. La obra se paraliza, comunicáis el hallazgo al Ayuntamiento y se amplía el perímetro de actuación para llevar a cabo la excavación arqueológica del área cercana a la estela. Las evidencias materiales que han aparecido hasta ahora son:

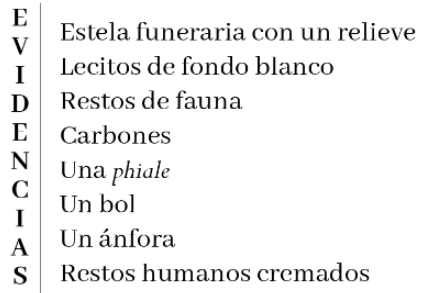

\begin{tabular}{|l|}
\hline \multicolumn{1}{|c|}{ ¡Recordad! } \\
Sois vosotros/as quienes "conocéis” o no: \\
- Ll relieve de la estela y los motivos decorativos de las \\
cerámicas, si los tienen, así como su conservación \\
- La especie/edad/partes anatómicas de los restos de fauna \\
- La edad/género de los restos humanos \\
- Así como otros resultados de análisis varios
\end{tabular}

Problema

Si bien la extensión de la necrópolis está todavía por confirmar, el Ayuntamiento de la ciudad os pide que acudáis a una rueda de prensa en la que se informe a la ciudadanía sobre el hallazgo.

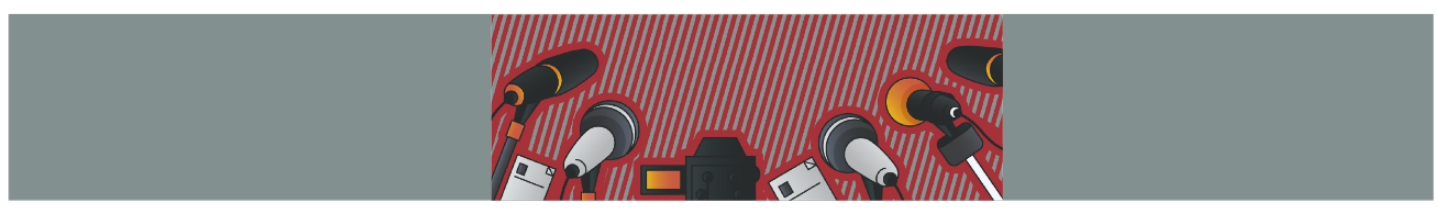

La prensa os planteará, entre otras, las siguientes preguntas:

1- ¿Cuál es el hallazgo más importante? ¿Por qué?

2- ¿Solo hay una tumba? ¿Podría haber más?

3- ¿Cómo sería el ritual funerario asociado a esta tumba?

4- ¿Qué ofrendas se hacían en las visitas posteriores?

Recordad que, si bien cada uno tenéis una
especialidad, sois un equipo y deberéis llegar
a un acuerdo antes de contestar a las
preguntas. Uno/a de vosotros/as será el
portavoz del equipo (director/a de la
excavación).

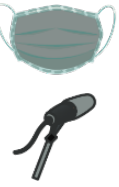

*Debido a la pandemia, la rueda de prensa se realizará por videoconferencia. Aun así, podréis utilizar vuestras manos para reconstruir la gestualidad de las prácticas rituales.

*¿Qué otras preguntas haríais si formarais parte del equipo de periodistas?

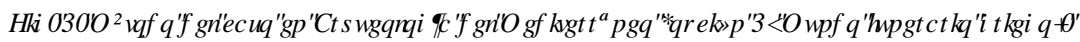




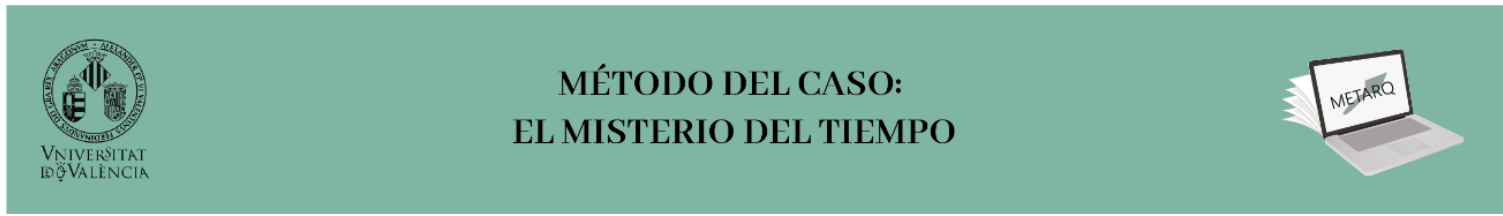

\section{Contexto}

Como cada semana, os dirigís a la oficina central para que os asignen una misión. Sin embargo, esta semana será distinta. Por primera vez el ministerio precisa de vuestros conocimientos como arqueólogas y arqueólogos. ¿Pensabais que nunca aplicaríais vuestra formación universitaria en este ministerio? ¡Pues el día ha llegado! Os notifican la existencia de una anomalía en el transcurso de una excavación arqueológica en una necrópolis ibérica. Ilasta la fecha, la excavación había transcurrido sin problemas. La mayoría de las tumbas localizadas y excavadas eran "normales", la estratigrafía tenía sentido y la datación relativa de las tumbas había sido bastante sencilla. Sin embargo, el ajuar hallado en esta tumba no es para nada normal. Por eso mismo, la directora de la excavación, quien fue funcionaria del ministerio hace unos años, se ha puesto en contacto con vosotros.

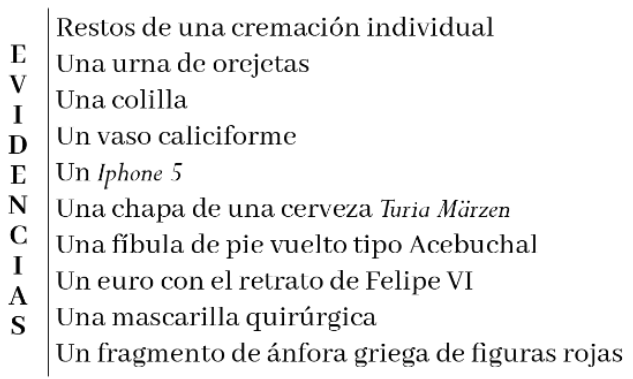

\section{Algunas pistas...}

Figuras rojas: a partir de 530 a.C.

- Datación C14 de los restos humanos: ca 500-490 BC - Fíbula tipo Acebuchal (ver Argente Oliver, 1987) - Urna de orejetas

(ver Mata y Bonet 1992; López Bravo, 2002)

* Podéis consultar Google para orientaros en la cronología del resto de objetos

\section{Problema}

El sedimento no parece mostrar evidencias de haber sido alterado, ni la tumba expoliada. En este tipo de contextos, es posible que existan alteraciones derivadas de procesos post-deposicionales naturales. Sin embargo, este caso es distinto... Está claro que ha habido un viajero del tiempo no registrado que ha tenido un descuido en el momento de deposición de los restos incinerados del difunto... Antes de que se filtre la noticia a la prensa, vosotros y vosotras, como patrulla especializada en contextos arqueológicos, debéis viajar en el tiempo al momento concreto de la incineración, averiguar los eventos que han podido desembocar en la intromisión de ciertos objetos y solucionar el error.

El Ministerio del tiempo os planteará, entre otras, las siguientes preguntas: 1. ¿А qué año o periodo aproximado viajaréis?

Teniendo en cuenta los materiales arqueológicos ¿Qué cronología relativa tendría la tumba?

\section{2. ¿Qué puerta/s escogeréis para solucionar el problema?}

Teniendo en cuenta las intrusiones identificadas ¿En qué horquilla cronológica nos movemos? ¿De qué fecha a qué fecha se pudo producir?

\section{3. ¿Qué ha podido pasar?}

Utilizando los conceptos que habéis aprendido en clase sobre datación, junto con los datos que habéis obtenido en vuestra investigación y con un poco de imaginación, debéis reconstruir el problema, adelantaros al acontecimiento y solucionarlo.
Recordad que, si bien cada uno podéis buscar la cronología de un objeto, sois un equipo y deberéis llegar a un acuerdo antes de contestar a las preguntas. Uno/a de vosotros/as será el/la portavoz del equipo (responsable de la patrulla).

\section{| BUENA SUERTE !}

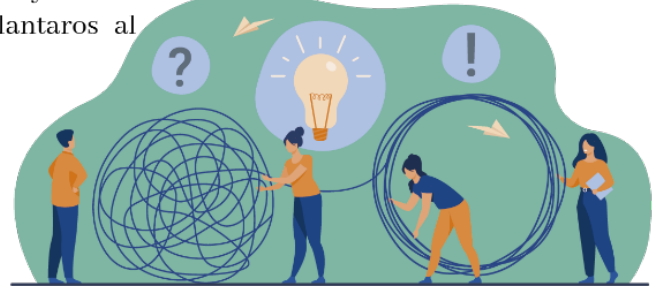

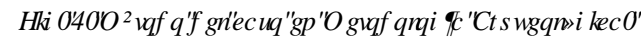




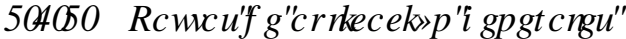

La puesta en práctica de ambos casos no precisaba de una gran variedad de recursos (ver tabla 1). Si bien ambos eran distintos, la aplicación fue similar. Sin embargo, siguiendo las orientaciones de la autoevaluación del profesorado y la retroalimentación del estudiantado durante el primer cuatrimestre, incorporamos ciertas mejoras para la aplicación del caso en el segundo cuatrimestre (asignatura de Metodología Arqueológica).

\begin{tabular}{|c|c|c|c|c|}
\hline Asignatura & Conceptos & Procedimientos & Actitudes & Recursos necesarios \\
\hline $\begin{array}{c}\text { Arqueología } \\
\text { del } \\
\text { Mediterráneo } \\
\text { (1er cuatrimestre) }\end{array}$ & $\begin{array}{l}\text { - Prácticas rituales } \square \\
\text { - Rituales funerarios } \square \\
\text { - Inhumación/cremación } \square \\
\text { - Ajuar funerario } \square \\
\text { - Metodología } \\
\text { arqueológica } \square \\
\text { - Arqueología sensorial } \square \\
\text { - Seguimiento } \\
\text { arqueológico }\end{array}$ & $\begin{array}{l}\text { Clases teóricas y } \\
\text { clases prácticas, } \\
\text { combinadas con } \\
\text { trabajo individual } \\
\text { y cooperativo en } \\
\text { clase (virtual o } \\
\text { presencial), } \\
\text { supervisado por } \\
\text { el profesorado }\end{array}$ & \multirow{2}{*}{$\begin{array}{l}\text { - Cooperación } \\
\text { - Diálogo } \\
\text { entre iguales } \\
\text { - Trabajo en } \\
\text { equipo } \\
\text { - Organización } \\
\text { - Toma de } \\
\text { decisiones } \\
\text { - Expresión } \\
\text { oral } \\
\text { - Capacidad } \\
\text { de análisis } \\
\text { - Autonomía }\end{array}$} & \multirow{2}{*}{$\begin{array}{l}\text { - Papel, bolígrafo y } \\
\text { algún dispositivo } \\
\text { electrónico (ordenador, } \\
\text { tablet o móvil) para } \\
\text { poder consultar } \\
\text { información de } \\
\text { referencia (presencial) } \\
\text { - Dispositivo electrónico } \\
\text { con conexión a internet } \\
\text { (\%DFNERDG] } \\
\text { \&RCDERUAAlvirtual) } \\
\text { *Aun así, se potencia el } \\
\text { debate frente a la } \\
\text { consulta de información } \\
\text { complementaria } \\
\text { (aconsejando que esta } \\
\text { consulta se realice en } \\
\text { última instancia) }\end{array}$} \\
\hline $\begin{array}{l}\text { Metodología } \\
\text { Arqueológica } \\
\left(2^{\circ} \text { cuatrimestre }\right)\end{array}$ & $\begin{array}{l}\text { - Métodos de datación } \square \\
\text { - Datación absoluta } \\
\text { - Datación relativa } \\
\text { - Tipología } \\
\text { cerámica/fíbulas } \square \\
\text { - Arqueología funeraria } \square \\
\text { - Alteraciones/ } \\
\text { fenómenos } \\
\text { - } \text { postdeposicionales } \square \\
\text { - } \text { arqueológica } \square \\
\text { - Prospección } \\
\text { arqueológica } \square \\
\text { [ }\end{array}$ & $\begin{array}{l}\text { Clases teórico- } \\
\text { prácticas, } \\
\text { combinadas con } \\
\text { trabajo individual } \\
\text { y cooperativo en } \\
\text { clase (virtual o } \\
\text { presencial), } \\
\text { supervisado por } \\
\text { el profesorado }\end{array}$ & & \\
\hline
\end{tabular}

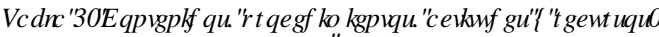

En ambos casos, fue vital el trabajo en equipo, tanto presencial como en línea, a través de \%OFNERDCT \&RCDERLWH Esta herramienta de videoconferencias permite compartir el contenido del ordenador y utilizar una pizarra virtual para interactuar, facilitando así el aprendizaje en línea síncrono y asíncrono. Es cierto que su aplicación efectiva todavía debe superar ciertas barreras (Alokluk, 2018). Sin embargo, cuenta con herramientas muy útiles, como la opción de crear grupos aleatorios posibilitando así el aprendizaje colectivo, también en línea.

Centrándonos en su aplicación específica, debemos indicar que esta fue distinta entre los grupos de Arqueología del Mediterráneo. En los grupos A y C fue 100\% presencial (se repitió en dos semanas consecutivas con una temática ligeramente distinta). En el grupo B se realizó de forma híbrida (algunos estudiantes estaban en el aula y otros estaban en casa, trabajando en grupos presenciales y grupos creados a través de \%DFNERDCF\&RCDERLWHlsupervisados todos por el profesorado). Este último procedimiento fue el que seguimos también en los grupos de Metodología Arqueológica. Evidentemente, en ambas asignaturas existió un paso previo a la actividad en la que el profesorado expuso los conceptos básicos necesarios. En el caso de Arqueología del Mediterráneo, se explicaron las características básicas de las prácticas funerarias en el mundo griego y romano, con ayuda de reconstrucciones $3 \mathrm{D}$ y otro tipo de recursos audiovisuales (1.30 $\mathrm{h}$ de clase teórica en los grupos A y C y 45 minutos durante las clases prácticas en el grupo B). En el caso de Metodología Arqueológica, se abordó el tema del tiempo y los métodos de datación, con material y ejemplos de apoyo como el vídeo grabado para este tema ${ }_{6}(1$ sesión y media de las clases teóricas: total

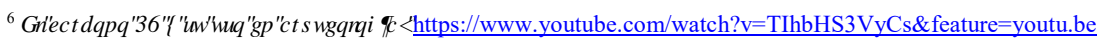


2.30 h). Además, de manera complementaria, se introdujo el tema de Arqueología funeraria para que el estudiantado comprendiera conceptos clave como ajuar funerario, cremación, inhumación... (media sesión de teoría: $45 \mathrm{~min}$.).

Durante las clases previas a la actividad se motivó la asistencia del estudiantado, explicándoles que se realizaría una práctica innovadora en la que se aplicarían los conocimientos adquiridos en las clases previas. Además, a través del Aula Virtual y de las redes sociales del Proyecto de Innovación Docente (@metarq ${ }^{7}$ se recordó la actividad días antes de la clase, a través de WRUH y publicaciones en el muro/hilo de ,QMDJUP/7ZLWU(fig. 3).

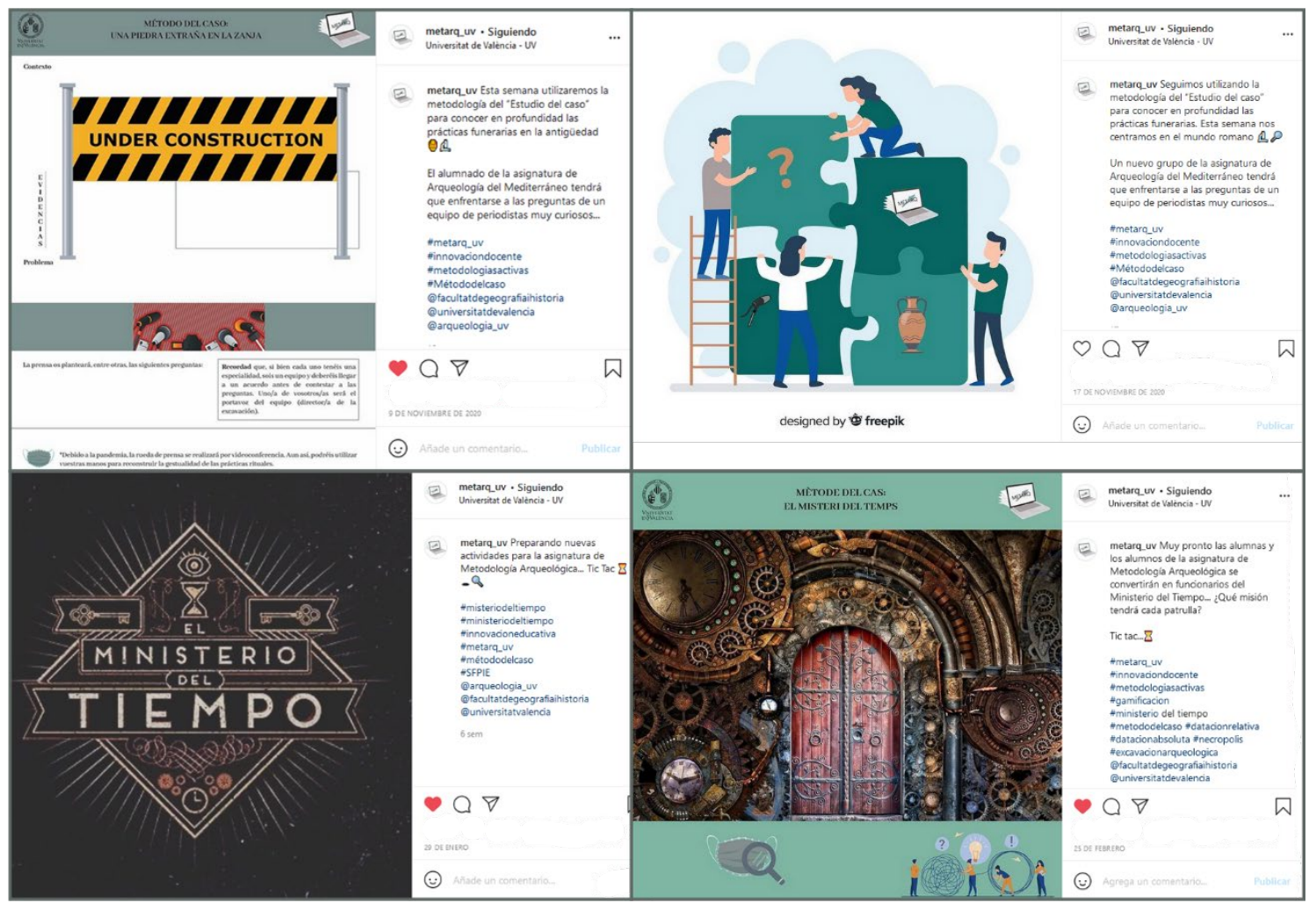

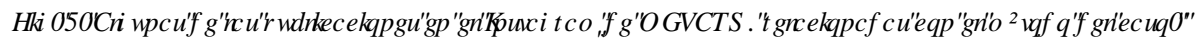

El siguiente paso fue la aplicación del método en sí, en clases prácticas de 45 minutos para Arqueología del Mediterráneo y en horario de clases teóricas $(45+45$ minutos $)$ para Metodología Arqueológica. Para ello seguimos, a grandes rasgos, los pasos que se observan en la tabla 2.

\footnotetext{
${ }^{7}$ https://www.instagram.com/metarq uv/?hl=es; https://twitter.com/metarq?lang=es
}

(cc) BY-NC-ND 2021, Universitat Politècnica de València 


\begin{tabular}{|c|c|c|c|c|}
\hline & Paso 1 & Paso 2 & Paso 3 & Paso 4 \\
\hline$\overbrace{0}^{\stackrel{0}{0}}$ & $\begin{array}{c}\text { Explicación de } \\
\text { la actividad }\end{array}$ & $\begin{array}{c}\text { Trabajo en pequeños grupos } \\
\text { (4-5 personas) }\end{array}$ & $\begin{array}{l}\text { Trabajo en } \\
\text { gran grupo } \\
\text { (debate) }\end{array}$ & $\begin{array}{l}\text { Reflexiones finales } \\
\text { (profesorado) }\end{array}$ \\
\hline 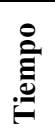 & $5 \mathrm{~min}$ & $30 \mathrm{~min}$ & 10-30 min* & $5-10$ min* \\
\hline 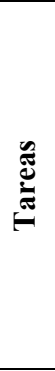 & $\begin{array}{l}\text { Explicación de } \\
\text { la mecánica de } \\
\text { la actividad, el } \\
\text { contexto y los } \\
\text { objetivos } \\
\text { principales (ver } \\
\text { figs. } 1 \text { y } 2 \text { ) }\end{array}$ & $\begin{array}{l}\text { - } \\
\text { Elección de portavoz } \\
\text { Elección de especialistas } \\
\text { (Arqueología del Mediterráneo) } \\
\text { /División de tareas de búsqueda } \\
\text { cronológica (Metodología } \\
\text { Arqueológica) } \\
\text { - } \begin{array}{l}\text { Debate en pequeños grupos las } \\
\text { soluciones y respuestas a las } \\
\text { preguntas planteadas y otras } \\
\text { posibles preguntas }\end{array} \\
\end{array}$ & $\begin{array}{l}\text { Se comparten } \\
\text { y discuten las } \\
\text { respuestas y } \\
\text { resultados } \\
\text { (portavoces } \\
\text { de cada } \\
\text { grupo) }\end{array}$ & $\begin{array}{ll}\text { - } & \text { Síntesis } \\
\text { - } & \text { Puntos positivos } \\
\text { - } & \text { Carencias (a mejorar) }\end{array}$ \\
\hline
\end{tabular}

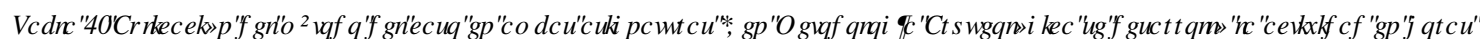

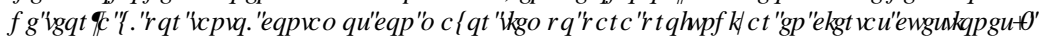

El segundo paso es distinto en cada asignatura (ver tabla 2). En Arqueología del Mediterráneo las primeras tareas de este paso son más complejas, ya que deben recordar los conceptos aprendidos en el curso anterior (asignatura de Metodología Arqueológica) para poder elegir los especialistas. En un primer momento no se les da ninguna orientación. Sin embargo, la supervisión del profesorado permite que en aquellos grupos en los que tan solo lleguen a extraer dos o tres especialistas se potencie la colaboración intergrupal y se pregunte al resto de los participantes qué más especialistas han escogido (esto les hace reflexionar sobre otras cuestiones que posiblemente no hayan tenido en cuenta): p.e. arqueozoología, antracología, antropología física, ceramología, epigrafía, ... En el ejemplo del Ministerio del Tiempo es más sencillo, ya que tan solo deben decidir quién busca la cronología de cada objeto, para después poner en común los datos recogidos en la bibliografía de referencia y las consultas a través de diversas páginas web.

El debate generado durante el tercer paso (ver tabla 2), también varía ligeramente entre asignaturas. En los grupos de Arqueología del Mediterráneo, sin previo aviso, se les informa de que la rueda de prensa comienza y de que son ellos y ellas mismas los que se convertirán en periodistas. El rol de arqueólogo/a y periodista va cambiando con cada pregunta. El profesorado se encarga de coordinar esta parte de la actividad, pudiendo también participar como periodista con nuevas preguntas que inciten al debate y la reflexión. Los portavoces de cada equipo de arqueólogos/as son los responsables de contestar (ver fig. 1). En los grupos de Metodología Arqueológica, el debate se realiza primero abordando una a una la cronología de los objetos hallados en la tumba (tanto de cronología ibérica, como de las intrusiones de elementos actuales producidas por el/la viajero/a en el tiempo). Un/a representante de cada grupo aporta una cronología para cada objeto y se debate entre toda la clase las diversas dataciones propuestas, así como las tipologías o los datos consultados (orientado por el profesorado). Finalmente, se debate la cronología de la tumba y del viaje en el tiempo y se dejan unos minutos para compartir las ideas de la tercera pregunta (ver fig. 2).

La actividad finaliza con la reflexión final del profesorado, centrada en el trabajo del estudiantado (cuarto paso) (ver tabla 2). El protagonismo del estudiantado está guiado, por tanto, por el profesorado que actúa como "faro", recuperando las preguntas y comentarios más interesantes, planteando preguntas que inciten a la reflexión y resumiendo los resultados clave de la discusión (Finkel, 2008). 


\section{Resultados}

Los resultados de aprendizaje adquiridos durante esta actividad se ven reflejados en distintos aspectos, dependiendo del grupo, la asignatura y los sistemas de evaluación recogidos en la guía docente.

Debemos tener en cuenta que la evaluación de la asignatura de Arqueología del Mediterráneo se realiza principalmente a través de un examen final ( $80 \%$ de la nota). El otro $20 \%$ se centra en las actividades complementarias. En la prueba escrita, se incluyen tanto preguntas de desarrollo, relacionadas con la temática tratada en las clases teóricas, como preguntas cortas (imágenes y/o definiciones), relacionadas con las clases prácticas. Para valorar el interés y el aprendizaje generado con el método del caso, realizamos una pregunta en el examen final sobre el mundo funerario (grupos A y C). La valoración fue bastante positiva, ya que la mayoría del estudiantado escogió esta opción de pregunta (49 alumnos, 84\% del grupo C). Si bien el porcentaje en el grupo A fue la mitad (31 alumnos, 50\%), las respuestas aportadas en la opción relacionada con el mundo funerario fueron sustancialmente mejores, con respuestas mucho más elaboradas, razonadas y más alejadas de una simple explicación de los apuntes. A grandes rasgos, comparando los resultados de este curso con los de años anteriores, observamos que han comprendido mucho mejor y profundizado sobre ciertos aspectos que otros años no desarrollaban por completo.

En Metodología Arqueológica, con el objetivo de valorar los resultados de aprendizaje derivados directamente de la actividad y contando con la experiencia del primer cuatrimestre, decidimos preparar una plantilla básica a rellenar por el estudiantado. Para ello creamos una tarea voluntaria en el aula virtual y propusimos al estudiantado que entregara, al finalizar la clase, el informe del grupo a través del aula virtual. La motivación para realizar esta actividad escrita deriva de tres cuestiones clave:

1. El interés del propio estudiantado a demostrar por escrito el trabajo que habían realizado (observado durante la aplicación de la actividad en el primer cuatrimestre) $\square$

2. La posibilidad de analizar las respuestas y generar una retroalimentación previa a la entrega de la primera actividad individual ( $10 \%$ de la nota final de la asignatura $\left.{ }^{8}\right)$, relacionada con los métodos de datación, y mejorar así el resultado final.

3. La implicación del estudiantado al indicar que se valoraría positivamente la participación en la actividad, aunque no estuviera reflejado en los porcentajes finales.]

En general las respuestas presentadas en el documento de dos páginas, subido al aula virtual, son correctas, pero precisan de una justificación completa que indique de dónde extraen la información para datar los objetos arqueológicos y actuales en un momento u horquilla cronológica determinada. Aun así, la implicación ha sido muy alta, demostrando una comprensión general de la tarea y los métodos de datación. De manera anecdótica, es interesante indicar que las respuestas a la tercera pregunta (ver. Fig. 2) han sido muy ingeniosas, relacionando en algunas ocasiones el viaje en el tiempo con la solución de la pandemia.
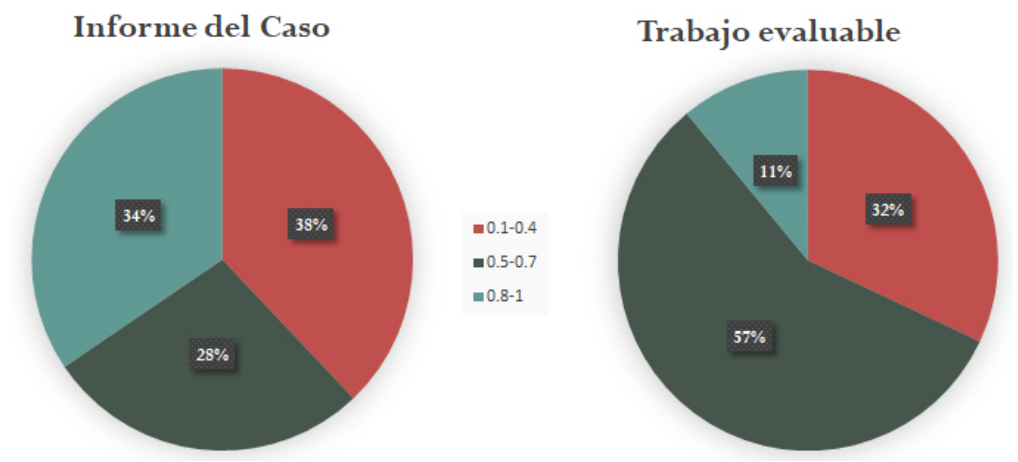

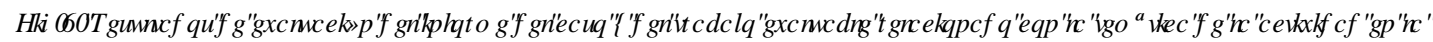

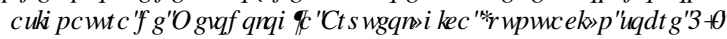

\footnotetext{
${ }^{8}$ La asignatura de Metodología Arqueológica sigue una evaluación continua: trabajos individuales (70\%) y un trabajo en grupo (30\%).
}

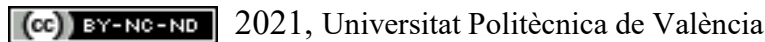

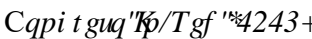


Tal y como se observa en la Figura 4, que considera los resultados de los tres grupos de Metodología Arqueológica en relación con el informe del método del caso (Fig.4, izquierda) y el ejercicio evaluable (Fig. 4, derecha), de los 152 alumnos que formaban 43 subgrupos de trabajo no han ejecutado del modo adecuado el informe del caso el 38\%. Sin embargo, el $28 \%$ de los grupos han trabajado de modo satisfactorio y el $34 \%$ de modo muy satisfactorio. Hay que subrayar que en el $38 \%$ de los grupos que no han trabajado adecuadamente, el $80 \%$ se trataba de grupos en línea y solo el $20 \%$ presencial, mientras que entre el $34 \%$ de los grupos que han trabajado muy satisfactoriamente esta diferencia se atenúa siendo el $40 \%$ de los grupos en línea y el $60 \%$ presencial los que logran llevar a cabo el ejercicio correctamente. Si observamos los resultados de la actividad evaluable (fig. 4, derecha), de los 152 alumnos no han llevado a cabo correctamente la actividad el $32 \%$, mientras que el $57 \%$ de los grupos han trabajado de modo satisfactorio y solo el $11 \%$ de modo muy satisfactorio. Aquí hay que subrayar que si el porcentaje de trabajos mal ejecutados es similar (32\%), disminuye ligeramente si lo comparamos con el informe del caso. Aunque no son actividades comparables, ya que el mecanismo de ejecución es distinto (y en algunas ocasiones ha sido causa de algunos malentendidos en el desarrollo del ejercicio evaluable), si comparamos los resultados con años previos observamos una ligera mejora. El hecho de abordar en grupo una tarea similar previa al trabajo evaluable parece haber mejorado la comprensión y la realización de la tarea.

Finalmente, es interesante indicar que en el grupo C de Metodología Arqueológica, en el cual se solicitó una autoevaluación propia de los grupos cuando entregaron el informe del caso, la mayoría del estudiantado ha sido consciente de su trabajo. Prueba de ello es la coincidencia de la nota del $80 \%$ de los grupos con la nota del profesorado, mientras que en el otro $20 \%$ la tendencia ha sido autoevaluarse con una nota inferior. Esta autoevaluación es una prueba más de la implicación y la participación activa del estudiantado en esta actividad.

(

\subsection{Opinión del estudiantado}

Con el objetivo de conocer la opinión del estudiantado sobre las actividades y herramientas de innovación implementadas durante el Proyecto de Innovación Docente METARQ, confeccionamos un formulario de * RRJOHpara cada uno de los grupos ${ }^{9}$. Si bien la mayoría de las preguntas coincidían, los enunciados se adaptaron teniendo en cuenta las actividades y herramientas que se habían implementado en cada grupo. En general, la retroalimentación de aquellas personas que contestaron la encuesta en los grupos del primer cuatrimestre (23 personas en el grupo A, 10 personas en el grupo B y 30 personas en el grupo C) fue bastante positivo. Si nos centramos en la valoración de la actividad que aquí nos ocupa, el método del caso, observamos que la opinión del estudiantado fue bastante positiva. En los tres grupos de Arqueología del Mediterráneo gran parte valoraron con un 5 de 5 esta actividad: 45-50\% (Fig. 5). En los grupos A y B, sí que estaban más familiarizados con esta herramienta, pero en el grupo $\mathrm{C}$, tan solo el 23,3\% habían asistido a otra clase del Grado de Historia en la que se aplicara esta metodología.

A la espera de realizar esta misma encuesta en los grupos de Metodología Arqueológica ( $2^{\circ}$ cuatrimestre), realizamos un breve cuestionario para conocer la opinión general del estudiantado sobre esta actividad en concreto (Grupo A: 30 respuestas, Grupo B: 28 respuestas y Grupo C: 35 respuestas). El resultado general muestra que la mayoría ha apreciado la actividad y que les gustaría que se aplicara tanto en otros temas, como en otras asignaturas ( $80 \%$ en el grupo A, $75 \%$ en el grupo B y $91,4 \%$ en el grupo C). Generalmente, han indicado que el caso les ha ayudado a mejorar la comprensión de ciertos conceptos (70\% en el grupo A, $64,2 \%$ en el grupo B y 100\% en el grupo C). Sin embargo, la valoración global de la actividad (0-5) no es tan positiva como la evidenciada en el primer cuatrimestre (ver fig. 5). Teniendo en cuenta las encuestas, es posible que esta valoración venga influenciada por la realización de la actividad de manera híbrida (presencial y virtual).

\footnotetext{
${ }^{9}$ Este formulario se adaptará para los grupos del segundo cuatrimestre (asignatura de Metodología Arqueológica) y se realizará durante el mes de mayo de 2021, contando así con datos comparativos completos.
} 


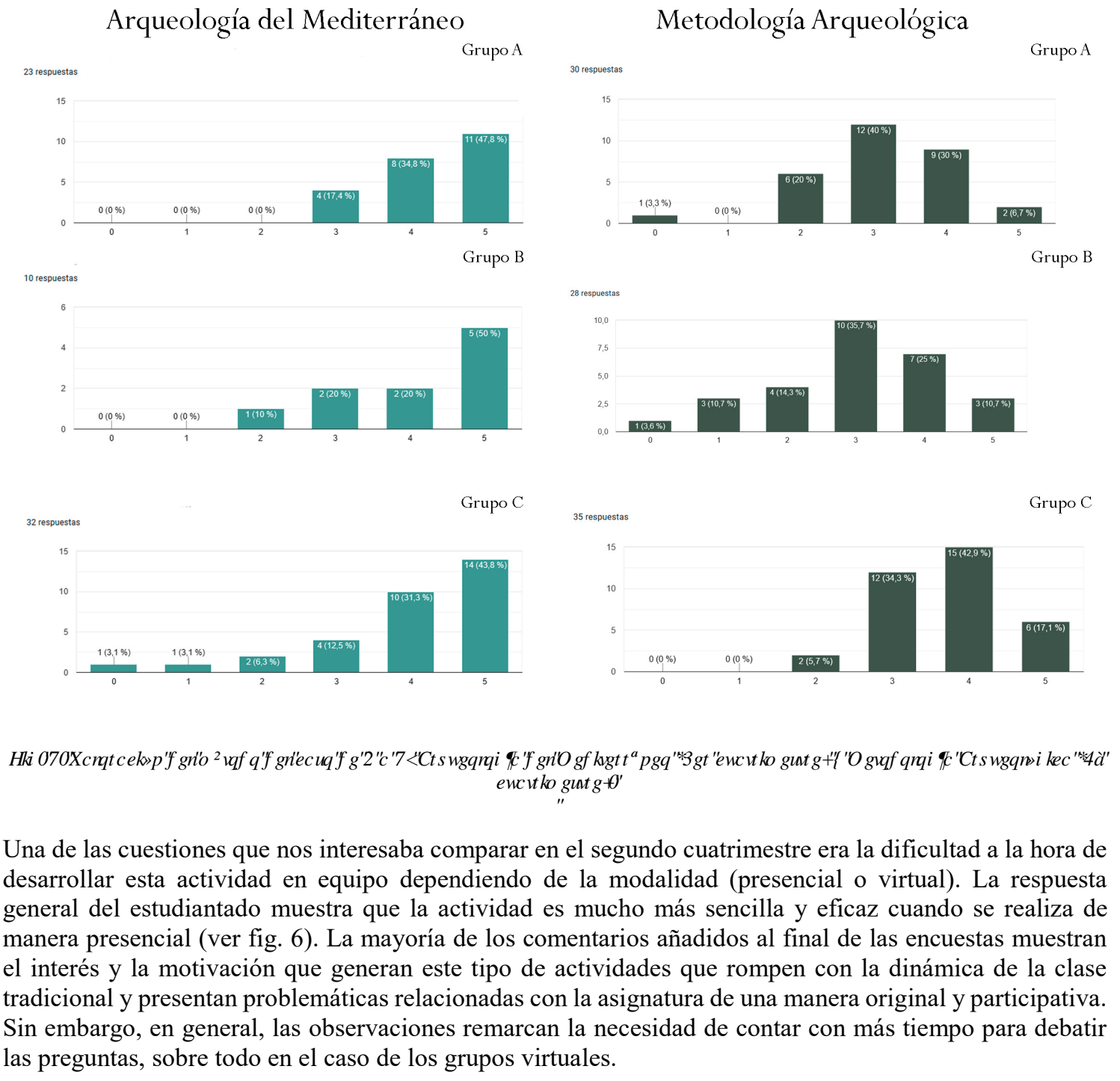

¿Te ha resultado fácil desarrollar esta actividad en equipo?

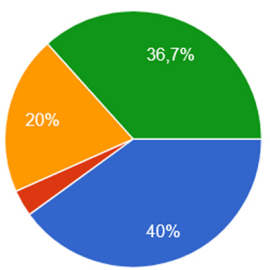

Grupo A

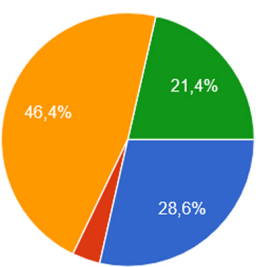

Grupo B

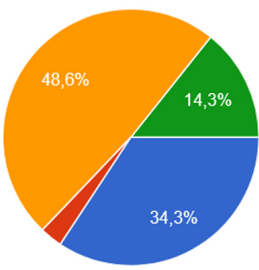

Grupo C
Sí (presencial)

No (presencial)

Sí (virtual)

No (virtual)

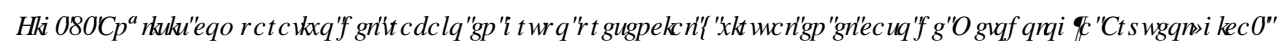




\section{Discusión}

Dentro del conjunto de actividades propuestas en el marco del Proyecto de Innovación Docente METARQ, el método del caso ha sido muy bien valorado por el profesorado, ya que es una herramienta que genera curiosidad y propicia el autoconocimiento. Sobre todo, valoramos el aprendizaje entre iguales y el trabajo en equipo, tanto virtual como presencial, así como la motivación general del estudiantado, la participación y la asistencia a clase.

Uno de los aspectos que consideramos más interesante es el debate que se genera, tanto en pequeños grupos como en el conjunto de la clase. Si bien todos los grupos cuentan con las mismas evidencias, los resultados obtenidos pueden ser muy dispares y siempre argumentados. Algunas respuestas pueden conseguirlas a partir de una simple búsqueda en internet o consulta de los artículos de referencia y otras pueden derivar de una decisión consensuada, basada en las evidencias. En el caso de Arqueología del Mediterráneo es muy importante la opinión de los "expertos y expertas", pudiendo variar en gran medida los resultados obtenidos. Estos "expertos/as" deciden algunas de las características del registro arqueológico, por ejemplo: ¿Se conservan restos óseos de la cremación que puedan ayudar a identificar el género o la edad del difunto? ¿La estela funeraria cuenta con algún motivo figurativo que informe sobre la vida del difunto? etc. En el caso de Metodología Arqueológica, sí que existían dos respuestas más o menos correctas (cronología de la tumba ibérica y horquilla cronológica en la que se pudo realizar el viaje en el tiempo). Sin embargo, la tercera pregunta dejaba vía libre a la imaginación, siempre basándose en datos arqueológicos, que añadía un plus de gamificación a la actividad.

Teniendo en cuenta que las clases prácticas en las que se implementó la actividad en Arqueología del Mediterráneo eran de 45 minutos (siguiendo la normativa COVID-19 de ventilación del aula), el tiempo disponible en estas clases fue muy limitado. Por ello, atendiendo al interés y la motivación del estudiantado demostrado durante el primer cuatrimestre, decidimos ampliar el tiempo de debate en Metodología Arqueológica, llevando a cabo la actividad durante las clases teóricas ( $45+45$ minutos). De este modo, se permitió profundizar en el debate de pequeños grupos, virtual y presencial, orientado por el profesorado (ver tabla 2).

Por lo que respecta a la participación, si comparamos las experiencias del primer y el segundo cuatrimestre, es evidente que la implicación es mucho más compleja cuando se realiza la actividad desde casa. Sin embargo, esta implicación varía dependiendo de los grupos. En el caso del grupo de $4^{\circ} \mathrm{B}$ (que realizaron la actividad de manera híbrida), la implicación del estudiantado que estaba en casa fue muy alta. De hecho, un representante de cada grupo abrió el micro en el tiempo de debate y se estableció una conversación fluida entre el estudiantado presencial y el virtual. Sin embargo, es cierto que el grupo B es el más reducido (un total de 41 alumnos). En Metodología Arqueológica, donde en todos los grupos se realizó de manera híbrida, la participación del estudiantado virtual fue más reducida. Esto puede deberse a que eran grupos más numerosos que, además, no llevaban tanto tiempo impartiendo clase híbrida con el profesorado implicado (ya que todo el mes de febrero fue completamente en línea). Sea como fuere, es evidente que debemos realizar ciertos cambios para adaptar aún más la actividad al estudiantado virtual y generar un debate fluido entre la totalidad del estudiantado.

\section{Conclusiones}

El ejemplo presentado aquí muestra que la práctica y el uso de casos potencia la motivación del estudiantado y la participación en clase. La mayoría de los objetivos planteados por el equipo docente han sido cumplidos. Esta herramienta ha permitido mejorar la comprensión de ciertos conceptos, a través del trabajo autónomo y colaborativo, el diálogo y el aprendizaje entre iguales. Si bien, en cada grupo han existido ciertas personas que han tomado la iniciativa, se ha motivado el debate y la expresión de ideas de la totalidad de integrantes del grupo. Además, en ciertos casos, nos ha sorprendido gratamente la creatividad de algunos grupos para abordar los problemas planteados. El hecho de que exista una coordinación real entre los tres grupos de cada una de las asignaturas favorece la evaluación de la actividad y permitirá ajustar el método en cursos venideros. 
Sin embargo, para conseguir mejorar la aplicación de este tipo de metodologías activas es necesario una autoevaluación continua y compartida por parte del equipo docente implicado, teniendo siempre en cuenta la opinión del estudiantado. Indicamos a continuación, aquellos elementos que precisan mejoras o cambios para próximos cursos:

- Solicitar la entrega de un documento escrito al finalizar la actividad (como se ha hecho en los grupos del $2^{\circ}$ cuatrimestre) y proponer una autoevaluación del estudiantado (como se ha hecho en el grupo $\mathrm{C}$ de Metodología Arqueológica) para que el estudiantado sea más consciente de su aprendizaje.

- Simplificar las preguntas en el ejemplo de Misterio del Tiempo, ya que, sobre todo, la pregunta número dos dificultaba la comprensión (ver fig. 2).

- Intentar, en la medida de lo posible, que la actividad se realice presencialmente o ampliar en su defecto el tiempo disponible para el debate en los grupos virtuales.

- Combinar el método del caso con la metodología del 3X]] y Gómez, 2010).

- Diseñar más casos para poner en práctica otros temas de la asignatura y proponer al final del curso una actividad en la que sean los propios alumnos/as quienes diseñen un caso y lo presenten a sus compañeros/as para que lo resuelvan.

- Modificar el ejercicio evaluable para que sea similar al ejercicio realizado en clase y evitar así posibles confusiones.

Estas son tan solo algunas ideas que esperamos aplicar en el futuro para seguir mejorando nuestra práctica docente y, en definitiva, conseguir una mayor motivación del estudiantado y una mejora del proceso de enseñanza-aprendizaje.

\section{Referencias}

ALOKLUK, J.A. (2018). "The effectiveness of Blackboard System: uses and limitations in information management" en , QWHODJ HWQRRP DMRQO DQDJHP HQW19, p. 133-149. DOI: 10.4236/iim.2018.106012

BAETEN, M., DOCHY, F. y STRUYVEN, K. (2013). "The effects of different learning environments on students' motivation for learning and their achievement" en \%ULMK-RXLODORIL( GXFDMRQDOB VFKRQRJ। 83, p. 484-501. DOI: http://doi.org./10.1111/j.2044-8279.2012.02076

BAO, W. (2020). "COVID-19 and online teaching in higher education: A case study of Peking University" en $+X P D Q$ \% KDIRUDQGF( P HJ IQJП7FKQRQRIHHI2, p. 13-15. DOI: https://doi.org/10.1002/hbe2.191

COUSIN, G. (2005). "Case Study Research" en -RXLQDORI* HRUSKKILIQ+LJKHU( GXFDMRQ 29, p. 421-427. DOI: https://doi.org/10.1080/03098260500290967 [

FINKEL, D. (2008). ' DUFCDHFRQ(D)ERFDFHUDD. Valencia: PUV.

GALLELlO, G., MACHAUSE LÓPEZ, S. y DiEZ CASTILlo, A. (2020). "Respuesta docente frente a la pandemia de la COVID-19: el uso de Blackboard y Flipped Teaching en la asignatura de Metodología Arqueológica" en 9,

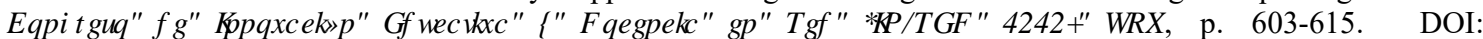
http://dx.doi.org/10.4995/INRED2020.2020.12012

GRANDON GIL, T. (2011). , QRLP IQ [Z LKIUKH\&DMHO HKRGIDJXIGHUR]FDMHP HKRG55 HMDFK, Santa Rosa (CA): Informing Science Press.

GERRING, J. (2007). “Is There a (Viable) Crucial-Case Method?” en \&RP SDWMYH3 RQMFDO6WGHМ40, p. $231-253$. DOI: https://doi.org/10.1177/0010414006290784

HARLAND, T. (2014). "Learning about case study methodology to research higher education" en + LJKHU( GXFDMRQ 5HMHFKD [' HHHRSP HQW33, p. 1113-1122. DOI: https://doi.org/10.1080/07294360.2014.911253

KILBANE, C, THEROUX, J., SUlEJ, J., BISSON, B., HAY, D. y BOYER, D. (2004). “The Real-Time Case Method: Description and Analysis of the First Implementation" en , QQRYDUYH + IJKHU ( GXFDMRQ 29, p. 121-135. DOI: https://doi.org/10.1023/B:IHIE.0000048794.27286.7c 
KOÇ, H. (2018). “Teaching Geography in Higher Education: A Case Study of Problem-Based Learning” en 5HIHZ

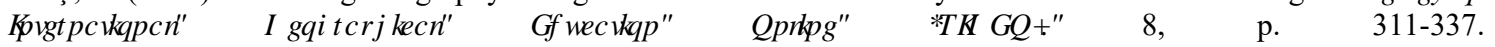
http://www.rigeo.org/vol8no2/Number2Summer/RIGEO-V8-N2-7.pdf

KREBER, C. (2001). "Learning Experientially through Case Studies? A Conceptual Analysis" en 7HDFKQJ $\amalg Q+I J K H \square$ ( GXFDURQ6, p. 217-228. DOI: https://doi.org/10.1080/13562510120045203

LARU, J., NÄYKKI, P. y JÄRVELÄ, S. (2012). "Supporting small-group learning using multiple Web 2.0 tools: A case study in the higher education context" HQ ,QUHQHW DQG + IJKH】 ( GXFDURQ 15, p 29-38. DOI: https://doi.org/10.1016/j.iheduc.2011.08.004

LIU, T-C., PENG, H., WU, W. y LIN, M. (2009). "The Effects of Mobile Natural-science Learning Based on the 5E Learning Cycle: A Case Study” en - RXLQDORIL GXFDMRQDOTHFQRQRJ I I6RFIHM] 12, p. 344-358.

MARTÍNEZ, J. y GÓMEZ, F. (2010). “La técnica puzzle de Aronson: descripción y desarrollo” en Arnaiz, P., ,Hurtado,

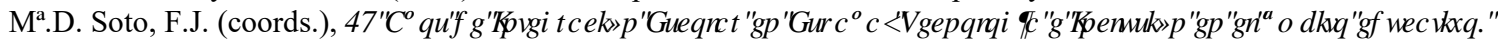
QDERWD $\square F R P$ XQUWUR. Murcia: Consejería de Educación, Formación y Empleo.]

MASSIS, A., y KOTLAR, J. (2014). "The case study method in family business research: Guidelines for qualitative

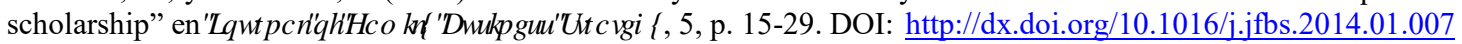

NKHOMA, M., SRIRATANAVIRIYAKUL, N. y LE QUANG, H. (2017). Using case method to enrich students' learning outcomes en \$FWH /HDQQJ] IQ + IJKHU (GXFDRQ 18, p. 37-50. DOI: https://doi.org/10.1177/1469787417693501

RASHID, Y., RASHID, A., WARRAICH, M.A., SABIR, S.S. y WASEEM, A. (2019). "Case Study Method: A Step-

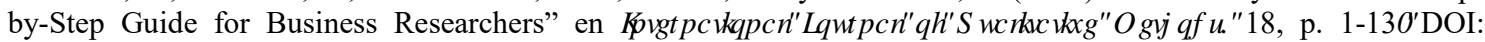
https://doi.org/10.1177/1609406919862424

SAFAPOUR, E.; KERMANSHACHI, S.; TANEJA, P. (2019): “A Review of Nontraditional Teaching Methods: Flipped Classroom, Gamification, Case Study, Self-Learning, and Social Media” HQ( GXFDMRQ6FHQFHV 9, p. 273. DOI: https://doi:10.3390/educsci9040273

SERVANT-MIKLOS, V.F.C. (2019). "The Harvard Connection: How the Case Method Spawned Problem-Based Learning at McMaster University" en +HDOW 3LRIHMRQV (GXFDMRQ 5, p. 163-171. DOI: https://doi.org/10.1016/j.hpe.2018.07.004

STEINER, G., y POSCH, A. (2006). "Higher education for sustainability by means of transdisciplinary case studies: an innovative approach for solving complex, real-world problems" en - RXLQDORIL\&ODQHU3 URGXFURQ 9, p. 877-890. DOI: https://doi.org/10.1016/j.jclepro.2005.11.054

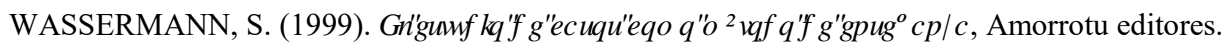

